\title{
Simulation of thin film flow around a cylinder on an inclined plane using the boundary element method
}

\author{
S. J. Baxter, H. Power, K. A. Cliffe \& S. Hibberd \\ The University of Nottingham, UK
}

\begin{abstract}
Steady Stokes flow driven by gravity down an inclined plane wall around a circular cylinder attached to the wall is considered. The effects of the cylinder are examined for various flow configurations. Values for the unit normal and curvature of the free surface are found using a Hermitian radial basis function interpolation. All free surface profiles indicate an upstream peak, followed by a trough downstream of the obstacle with the peak decaying in a "horseshoe" shaped deformation. Flow profiles are governed by four parameters; the plane inclination, the Bond number, the contact angle and the obstacle geometry.

Keywords: BEM, three-dimensional, thin film, Newtonian viscous flow, cylinder.
\end{abstract}

\section{Introduction}

Thin film flows down an inclined plane wall driven by gravity can often be modelled as an incompressible Stokes flow. Flows usually involve interaction with an obstacle either fully submerged of protruding through the film surface.

Early published works considering two-dimensional film flows over obstacles utilized a variety of techniques for the numerical procedures required to obtain solutions. An overview of these publications is presented in Blyth and Pozrikidis [1].

Hayes et al. [2] considered a three-dimensional steady, thin, viscous liquid film down an inclined plane driven by gravity and over small topographies. The lubrication approximation was used as the basis for their model and formulates a single linear inhomogeneous evolution equation. The free surface shape was then obtained by formation of the appropriate Green's function. 
The use of a direct boundary integral equation (BIE) for a three-dimensional Stokes flow analysis of film flow down an inclined plane and over an obstacle was first implemented by Pozrikidis and Thoroddsen [3] and later refined by Blyth and Pozrikidis [1]. The model in Pozrikidis and Thoroddsen [3] is based around the asymptotic limit where the size of a spherical obstacle is much smaller than the undisturbed film depth. Flows are shown to form an upstream peak and downstream trough with the peak decaying in the form of a "horseshoe". The extension of this work by Blyth and Pozrikidis [1] focuses on generalizing the earlier analysis to more arbitrarily sized obstructions. However, both simulations implement a linearization about the free surface deflection and thus the accuracy of significant deformations caused by large obstacles is unknown.

Numerical simulation of flow around obstacles has not been extensively considered. To the authors knowledge, the only applicable published work is Sellier [4]. This studies flow around a cylinder using the lubrication approximation, with the governing equations solved by the FEMLAB package. This approach is unable to impose a no slip boundary condition on the obstacle and is more relevant to far field displacements.

This paper uses the BIE formulation outlined by Blyth and Pozrikidis [1] to implement a modified analysis for film flows around large obstacles. A Hermitian radial basis function ( $\mathrm{RBF}$ ) is used for evaluation of the free surface terms required and is implemented as a replacement for previous finite difference approximations (FDAs) [1,3]. Elimination of the restriction that the free surface deformation is small $[1,3]$, allows accurate results for large obstacles.

\section{Mathematical formulation}

Figure 1 shows a two-dimensional schematic of a typical film flow of undisturbed thickness $H$ travelling down a plane inclined at angle $\alpha$ and around an obstacle attached to the plane. The inclined plane is referred to as $S_{w}$, the obstacle-fluid surface $S_{p}$ the disturbed free surface $S_{f}$, and the outward unit normal is denoted

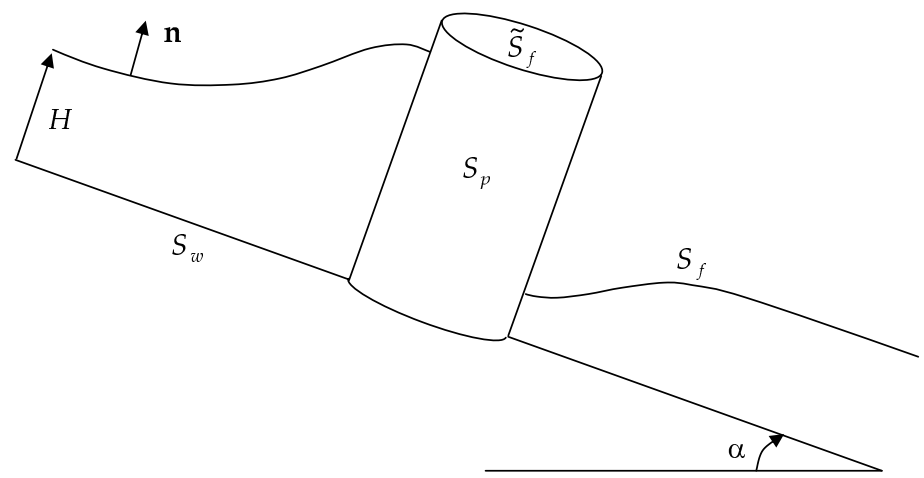

Figure 1: A cross section of a typical film profile. 
n. Finally the Cartesian co-ordinate system is aligned so that the $x_{1} x_{2}$-plane defines the undisturbed surface, with $x_{1}$ in the direction of the flow, and $x_{3}$ perpendicular to the undisturbed flow.

For convenience the physical variables are first non-dimensionalized. The undisturbed film height, $H$ is used for the reference length, the undisturbed free stream surface velocity, $U_{s}=\frac{H^{2} \rho g \sin \alpha}{2 \mu}$ (given by the Nusselt solution) for the reference velocity, and a viscous scaling $\frac{\mu U_{s}}{H}$ used for the tractions. The associated Bond number is given by,

$$
B o=\frac{\rho g H^{2} \sin \alpha}{\gamma},
$$

where $\gamma$ is the surface tension, $\rho$ the fluid density and $g$ the acceleration due to gravity. The inverse Bond number $B$ is defined by Blyth and Pozrikidis [1] using the mass flux and is related to eqn (1) by $B=\frac{\sin ^{1 / 3} \alpha}{B o}$.

The flow problem is governed by the usual incompressible equations for conservation of mass eqn (2) and steady Stokes flow eqn (3),

$$
\begin{aligned}
\frac{\partial u_{i}}{\partial x_{i}} & =0 \\
-\frac{\partial p}{\partial x_{i}}+\frac{\partial G}{\partial x_{i}}+\frac{\partial^{2} u_{i}}{\partial x_{j}^{2}} & =0
\end{aligned}
$$

where $G=-2\left(x_{3} \cot \alpha-x_{1}\right)$. The far field boundary conditions involve the flow velocity and pressure returning to undisturbed values far from the obstacle and the free surface deflection decaying to zero, i.e,

$$
\left.\begin{array}{c}
u_{i} \rightarrow u_{i}^{\infty} \\
p \rightarrow p^{\infty} \\
h \rightarrow 0 \\
\frac{\partial h}{\partial x_{1}} \rightarrow 0 \\
\frac{\partial h}{\partial x_{2}} \rightarrow 0
\end{array}\right\} \text { as } \mathbf{x} \rightarrow \pm \infty
$$

Boundary conditions must include no slip (zero velocity) on the wall and obstacle surface eqn (5), and a kinematic eqn (6) and dynamic eqn (7) condition applied on the free surface. These requirements can be conveniently expressed as

$$
\begin{gathered}
u_{i}=0 \quad \mathbf{x} \in S_{w} \cup S_{p}, \\
\frac{\partial x_{i}}{\partial t} n_{i}=u_{j} n_{j} \quad \mathbf{x} \in S_{f}, \\
f_{i}=\sigma_{i j} n_{j}=-\frac{4}{B o} \kappa n_{i} \quad \mathbf{x} \in S_{f},
\end{gathered}
$$


where $t$ is time, $f_{i}$ is the boundary traction, $\kappa$ is the curvature of the surface and $\sigma_{i j}$ is the stress tensor. Expressions for curvature and the stress tensor are

$$
\begin{aligned}
\kappa & =\frac{1}{2} \frac{\partial n_{i}}{\partial x_{i}}, \\
\sigma_{i j} & =-p \delta_{i j}+\left(\frac{\partial u_{i}}{\partial x_{j}}+\frac{\partial u_{j}}{\partial x_{i}}\right) .
\end{aligned}
$$

Finally, a contact angle $\theta$ between the free surface and the obstacle boundary must be specified and dependent on the fluid / obstacle properties. This is incorporated into the problem by

$$
\frac{\partial h}{\partial x_{i}} n_{i}=\tan \left(\frac{\pi}{2}-\theta\right),
$$

which is valid along the contact line.

In the absence of an obstacle, the governing equations are

$$
\begin{aligned}
\frac{\partial u_{i}^{\infty}}{\partial x_{i}} & =0, \\
-\frac{\partial p^{\infty}}{\partial x_{i}}+\frac{\partial G}{\partial x_{i}}+\frac{\partial^{2} u_{i}^{\infty}}{\partial x_{j}^{2}} & =0,
\end{aligned}
$$

and $u_{i}^{\infty}, p^{\infty}$ corresponds to the far field asymptotic case. In addition the no-slip boundary conditions for the asymptotic flow give $u_{i}^{\infty}=0$ for $\mathbf{x} \in S_{w}$, and solutions can be readily shown as

$$
\begin{aligned}
p^{\infty} & =-2 x_{3} \cot \alpha, \\
u_{i}^{\infty} & =\left(1-x_{3}^{2}\right) \delta_{i 1} .
\end{aligned}
$$

The asymptotic boundary traction is given by $f_{i}^{\infty}=\sigma_{i j}\left(p^{\infty}, u_{k}^{\infty}\right) n_{j}$, and using eqn (9) becomes

$$
f_{i}^{\infty}=2 x_{3}\left(\cot \alpha n_{i}-n_{3} \delta_{i 1}-n_{1} \delta_{i 3}\right) .
$$

Solutions eqn (13), eqn (14) govern the Stokes flow down an inclined plane in the absence of obstacles and are used as the basis for determining conditions for a disturbance flow due to the obstacle.

Disturbance velocities and pressures are denoted as $u_{i}^{\delta}=u_{i}-u_{i}^{\infty}$ and $p^{\delta}=$ $p-p^{\infty}$ respectively. Governing equations, obtained by comparing eqns (2)-(3) with eqns (11)-(12) are

$$
\begin{array}{r}
\frac{\partial u_{i}^{\delta}}{\partial x_{i}}=0, \\
-\frac{\partial p^{\delta}}{\partial x_{i}}+\frac{\partial^{2} u_{i}^{\delta}}{\partial x_{j}^{2}}=0 .
\end{array}
$$


To satisfy the far-field condition eqn (4), $u_{i}^{\delta}$ and $p^{\delta}$ are subject to the conditions

$$
\left.\begin{array}{l}
u_{i}^{\delta} \rightarrow 0 \\
p^{\delta} \rightarrow 0
\end{array}\right\} \text { as } \mathbf{x} \rightarrow \pm \infty
$$

Velocity conditions are found on the wall, $S_{w}$ and obstacle, $S_{p}$ and require

$$
\begin{aligned}
& u_{i}^{\delta}=0 \quad \mathbf{x} \in S_{w}, \\
& u_{i}^{\delta}=-u_{i}^{\infty} \quad \mathbf{x} \in S_{p} .
\end{aligned}
$$

In addition the associated disturbance boundary traction, $f_{i}^{\delta}$ is defined on the free surface $S_{f}$ from eqn (7) as

$$
f_{i}^{\delta}=-\frac{4}{B o} \kappa n_{i}-f_{i}^{\infty},
$$

where $f_{i}^{\infty}$ is given by eqn (14), and $\kappa$ is the curvature associated with the disturbed free surface.

\subsection{Boundary integral formulation}

The equations of Stokes flow for the disturbance quantities eqns (15)-(16) can be expressed exactly as an integral representation eqn (20),

$$
\begin{aligned}
c_{i j}\left(\mathbf{x}_{0}\right) u_{i}^{\delta}\left(\mathbf{x}_{0}\right)= & \frac{1}{8 \pi} \int_{S_{f} \cup S_{p}} G_{i j}^{*}\left(\mathbf{x}, \mathbf{x}_{0}\right) f_{i}^{\delta}(\mathbf{x}) \mathrm{d} S(\mathbf{x}) \\
& -\frac{1}{8 \pi} \int_{S_{f} \cup S_{p}} u_{i}^{\delta}(\mathbf{x}) T_{i j k}^{*}\left(\mathbf{x}, \mathbf{x}_{0}\right) n_{k}(\mathbf{x}) \mathrm{d} S(\mathbf{x}) .
\end{aligned}
$$

To close the obstacle a virtual surface is introduced and labeled $\tilde{S}_{f}$. For the closed domain $S_{p} \cup \tilde{S}_{f}$, the Stokes flow associated with the far field quantities $u_{i}^{\infty}$ and $p^{\infty}-G$ is satisfied by the integral representation eqn (21)

$$
\begin{aligned}
c_{i j}\left(\mathbf{x}_{0}\right) u_{i}^{\infty}\left(\mathbf{x}_{0}\right)= & -\frac{1}{8 \pi} \int_{S_{p} \cup \tilde{S}_{f}} G_{i j}^{*}\left(\mathbf{x}, \mathbf{x}_{0}\right)\left(f_{i}^{\infty}(\mathbf{x})+G n_{i}(\mathbf{x})\right) \mathrm{d} S(\mathbf{x}) \\
& +\frac{1}{8 \pi} \int_{S_{p} \cup \tilde{S}_{f}} u_{i}^{\infty}(\mathbf{x}) T_{i j k}^{*}\left(\mathbf{x}, \mathbf{x}_{0}\right) n_{k}(\mathbf{x}) \mathrm{d} S(\mathbf{x}) .
\end{aligned}
$$

The coefficient $c_{i j}\left(\mathbf{x}_{0}\right)$ takes a value 0 when $\mathbf{x}_{0}$ is outside the domain, $\frac{1}{2} \delta_{i j}$ when $\mathbf{x}_{0}$ is on the domain boundary and $\delta_{i j}$ when $\mathbf{x}_{0}$ is within the domain. Functions $G_{i j}^{*}\left(\mathbf{x}, \mathbf{x}_{0}\right), T_{i j k}^{*}\left(\mathbf{x}, \mathbf{x}_{0}\right)$ are the Lorentz-Blake Green's function for velocity and stress respectively satisfying zero velocity value at the inclined plane. Noting that $f_{i}^{\delta} \rightarrow$ 0 and $u_{i}^{\delta} \rightarrow 0$ as $\mathbf{x} \rightarrow \pm \infty$, the edges of the flow domain are omitted from eqn (20) as well. 
By a similar derivation to flow over an obstacle as described by Blyth and Pozrikidis [1], two BIE are derived, one collocated over the free surface $S_{f}$ and one over the obstacle-fluid surface $S_{p}$. The BIE for $\mathbf{x}_{0} \in S_{f}$ is,

$$
\begin{aligned}
\frac{1}{2} u_{j}^{\delta}\left(\mathbf{x}_{0}\right) & +\frac{1}{8 \pi} \int_{S_{f}} u_{i}^{\delta}(\mathbf{x}) T_{i j k}^{*}\left(\mathbf{x}, \mathbf{x}_{0}\right) n_{k}(\mathbf{x}) \mathrm{d} S(\mathbf{x}) \\
= & \frac{1}{8 \pi} \int_{S_{p}} G_{i j}^{*}\left(\mathbf{x}, \mathbf{x}_{0}\right) \tilde{f}_{i}(\mathbf{x}) \mathrm{d} S(\mathbf{x}) \\
& +\frac{1}{8 \pi} \int_{S_{f}} G_{i j}^{*}\left(\mathbf{x}, \mathbf{x}_{0}\right) f_{i}^{\delta}(\mathbf{x}) \mathrm{d} S(\mathbf{x}) \\
& +\frac{1}{8 \pi} \int_{\tilde{S}_{f}} G_{i j}^{*}\left(\mathbf{x}, \mathbf{x}_{0}\right)\left(f_{i}^{\infty}(\mathbf{x})+G n_{i}(\mathbf{x})\right) \mathrm{d} S(\mathbf{x}) \\
& -\frac{1}{8 \pi} \int_{\tilde{S}_{f}} u_{i}^{\infty}(\mathbf{x}) T_{i j k}^{*}\left(\mathbf{x}, \mathbf{x}_{0}\right) n_{k}(\mathbf{x}) \mathrm{d} S(\mathbf{x}) \quad \mathbf{x}_{0} \in S_{f},
\end{aligned}
$$

where $\tilde{f}_{i}(\mathbf{x})=f_{i}(\mathbf{x})+G n_{i}$. For $\mathbf{x}_{0} \in S_{p}$ the BIE is derived as

$$
\begin{aligned}
\frac{1}{8 \pi} \int_{S_{p}} G_{i j}^{*}\left(\mathbf{x}, \mathbf{x}_{0}\right) \tilde{f}_{i}(\mathbf{x}) \mathrm{d} S(\mathbf{x})= & -u_{j}^{\infty}\left(\mathbf{x}_{0}\right)-\frac{1}{8 \pi} \int_{S_{f}} G_{i j}^{*}\left(\mathbf{x}, \mathbf{x}_{0}\right) f_{i}^{\delta}(\mathbf{x}) \mathrm{d} S(\mathbf{x}) \\
& +\frac{1}{8 \pi} \int_{S_{f}} u_{i}^{\delta}(\mathbf{x}) T_{i j k}^{*}\left(\mathbf{x}, \mathbf{x}_{0}\right) n_{k}(\mathbf{x}) \mathrm{d} S(\mathbf{x}) \\
& -\frac{1}{8 \pi} \int_{\tilde{S}_{f}} G_{i j}^{*}\left(\mathbf{x}, \mathbf{x}_{0}\right)\left(f_{i}^{\infty}(\mathbf{x})+G n_{i}(\mathbf{x})\right) \mathrm{d} S(\mathbf{x}) \\
& +\frac{1}{8 \pi} \int_{\tilde{S}_{f}} u_{i}^{\infty}(\mathbf{x}) T_{i j k}^{*}\left(\mathbf{x}, \mathbf{x}_{0}\right) n_{k}(\mathbf{x}) \mathrm{d} S(\mathbf{x}) \quad \mathbf{x}_{0} \in S_{p} .
\end{aligned}
$$

In summary the steady, gravity driven Stokes flow down an inclined plane around an obstacle is governed exactly by solutions satisfying the BIE's given in eqn (22) and eqn (23), the kinematic condition eqn (6) and the dynamic condition eqn (7), along with the far field equations eqn (4). As the obstacle protrudes through the free surface a contact line is present and the contact angle condition eqn (10) is also required.

\subsection{Finite free surface deflections and the radial basis functions}

A modification to the linearized approach of Blyth and Pozrikidis [1] using a Hermitian RBF allows accurate calculations of the free surface curvature and spatial derivatives. Further, the method also allows the incorporation of the free surface far field conditions and the contact angle condition. At any point on the free surface, the height is given by $h\left(x_{1}, x_{2}\right)$, and takes values $h^{i}$ at data point $i$ for a total 
of $N$ points. The surface can then be interpolated using a $\operatorname{RBF} \psi\left(\left\|\mathbf{x}-\boldsymbol{\xi}^{j}\right\|\right)$ where $j=1, \ldots, N$ and $\boldsymbol{\xi}^{j}$ are the $x_{1}, x_{2}$ co-ordinates of the data points $h^{j}$. Thin plate splines (TPS) was used for the radial basis function, and require the addition of a polynomial term of order $m-1$ to guarantee the invertibility of the interpolation matrix, where $m$ is specified within the thin plate spline.

The values of $\frac{\partial h}{\partial x_{1}}$ and $\frac{\partial h}{\partial x_{2}}$ can be constrained at a selected set of $n$ data points, whilst still defining $h$ on the original $N$ data points. A solution for $h$ can be obtained using the differential operators $\frac{\partial}{\partial x_{1}}$ and $\frac{\partial}{\partial x_{2}}$ but acting on the thin plate splines second argument $\boldsymbol{\xi}$, i.e. $\frac{\partial}{\partial \xi_{1}}$ and $\frac{\partial}{\partial \xi_{2}}$. Similar constraint conditions are imposed in the interpolation to satisfy the contact condition eqn (10).

To complete the computation for finite disturbances, the outward unit normal and mean curvature of the free surface are both required. Evaluation of the unit normal will require derivatives $\frac{\partial h}{\partial x_{1}}$ and $\frac{\partial h}{\partial x_{2}}$, with the mean curvature needing in addition, $\frac{\partial^{2} h}{\partial x_{1}^{2}}, \frac{\partial^{2} h}{\partial x_{2}^{2}}$, and $\frac{\partial^{2} h}{\partial x_{1} \partial x_{2}}$ on the surface, which are obtained by the radial basis interpolation.

\section{Numerical examples}

Figure 2 shows the obtained numerical result of the free surface mesh for flow around a cylinder of radius $a=2.0$. The flow was down a plane inclined at $\alpha=$

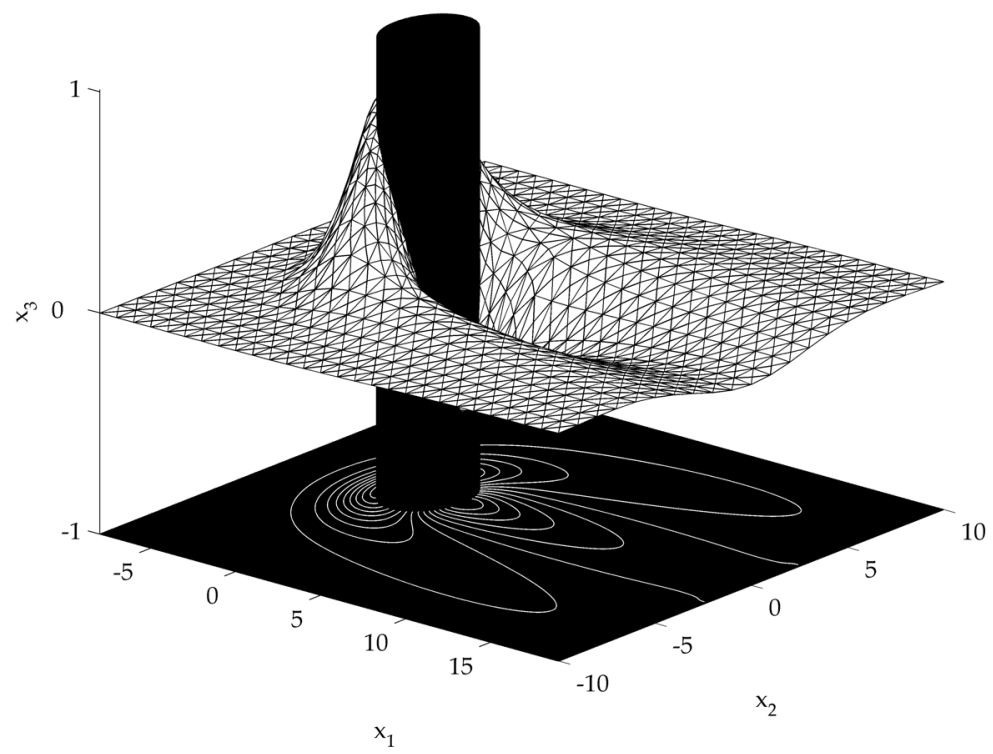

Figure 2: The free surface mesh and contours showing flow around a large cylinder of radius $a=2.0$. The plane is inclined at $\alpha=45^{\circ}$, the contact angle is set to $\theta=90^{\circ}$ and the inverse Bond number is 1 . 
$45^{\circ}$ and the film has an inverse Bond number of $B=1$. The contact angle at the cylinder walls was constrained to $\theta=90^{\circ}$.

Variations of the inverse Bond number $B$ was considered for flow down a plane inclined at $\alpha=45^{\circ}$ around a cylindrical obstacle of radius $a=0.5$. The free surface - obstacle interface was modelled by a contact angle of $\theta=90^{\circ}$. Increasing the inverse Bond number, corresponding to a raising of surface tension forces, was shown to flatten the centre line profiles. Variations of plane inclination angles $\alpha$ were also considered. Flow with an inverse Bond number $B=1$ and around a cylindrical obstacle of radius $a=0.5$ was considered. The free surface - obstacle interface was modelled by a contact angle of $\theta=90^{\circ}$. Increasing the inclination angle resulted in a raising of the peak before the obstacle.

Figure 3 indicates the smoothed centre line solutions for flow around a range of cylinder radii. Flow is for an inverse Bond number $B=1$ and down a plane inclined at $\alpha=45^{\circ}$. The obstacle was chosen neutrally wetting, (i.e. the contact angle was $\theta=90^{\circ}$ ). Increasing the cylinder radius results in a raising of the peak and deepening of the trough heights both before and behind the obstacle. Figure 4 illustrates the contact line profile around the cylinder wall for the range of cylinder radii depicted in figure 3 . Interestingly intersection of the contact line profiles occurs at an approximately fixed angular position along the cylinder, between 0.94-0.98 radians upstream of the position $x_{1}=a$.

Figure 5 indicates the smoothed centre line solutions for flow around a cylinder of radius 0.5 . Flow is for an inverse Bond number $B=1$ and down a plane

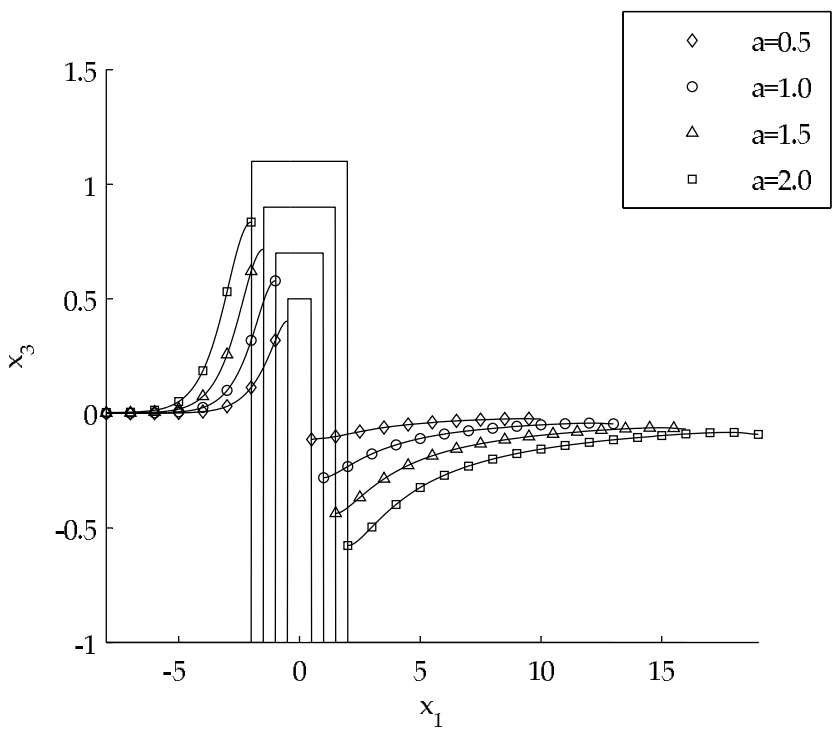

Figure 3: Centre line solutions for free surface deflections around a cylinder of varying radius, with inverse Bond number $B=1$ and down a plane inclined at $45^{\circ}$. The contact angle at the cylinder wall is $\theta=90^{\circ}$. 


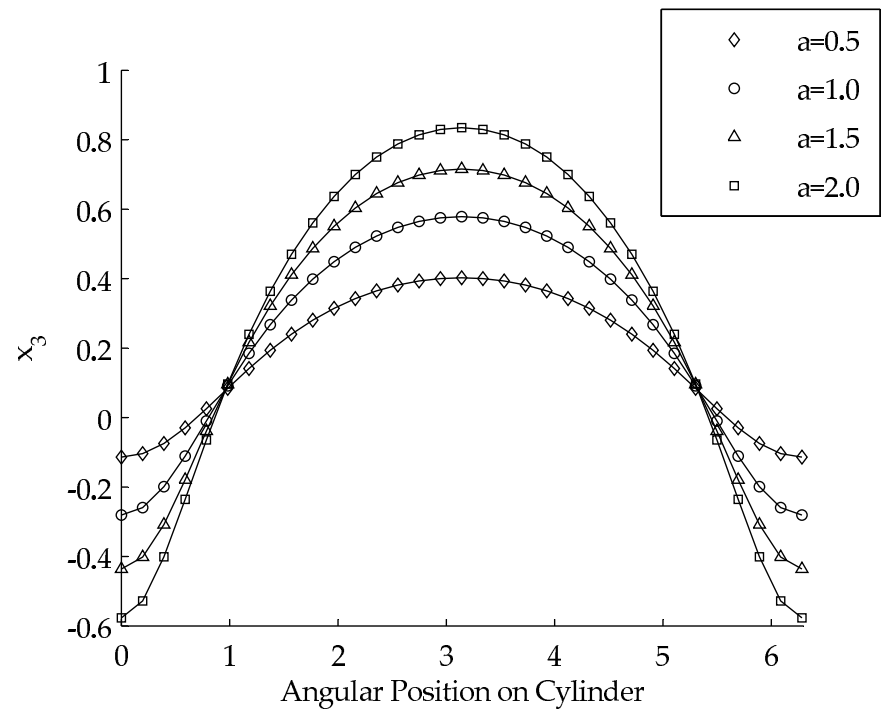

Figure 4: Contact line solutions for free surface deflections around a cylinder of varying radius, with inverse Bond number $B=1$ and down a plane inclined at $45^{\circ}$. The contact angle at the cylinder wall is $\theta=90^{\circ}$.

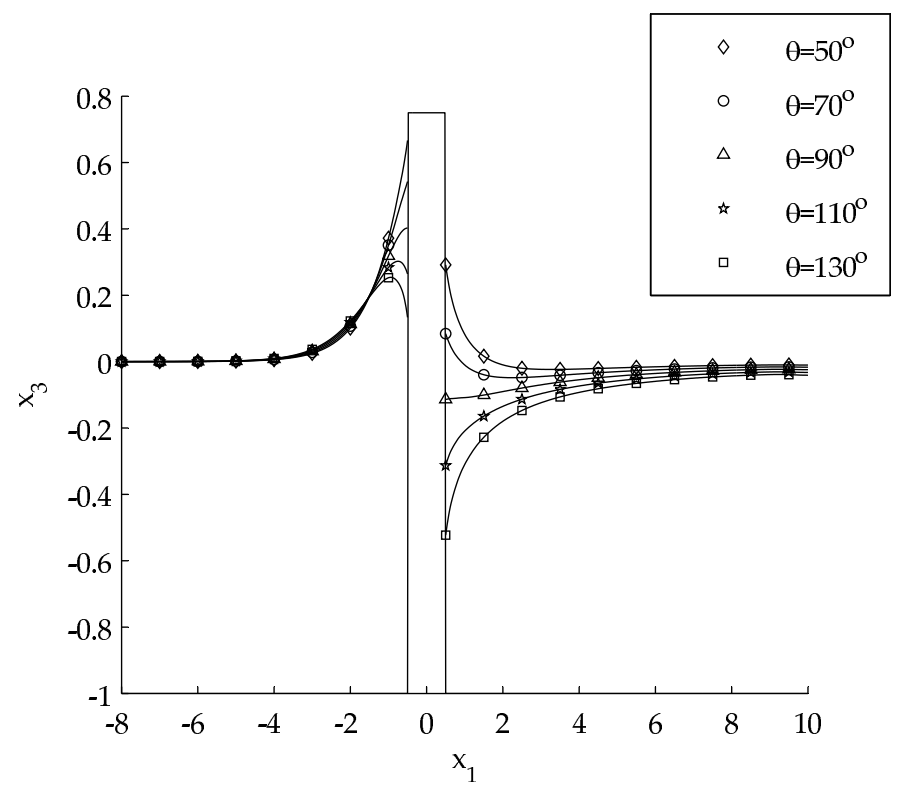

Figure 5: Centre line solutions for various contact angles at the cylinder wall. The free surface deflections are around a cylinder of radius $a=0.5$ on an inclined plane at $45^{\circ}$ and the flow has an inverse Bond number $B=1$. 
inclined at $\alpha=45^{\circ}$. The solutions show a range of wetting and non-wetting flow configurations corresponding to a range of contact angles $\theta$. Decreasing the contact angle results in a raising of the peak and trough heights both before and behind the obstacle.

\section{Conclusions}

Stokes flow down an inclined plane around a cylinder is considered and solutions found by the Boundary Element Method (BEM). Use of a global Radial Basis Function (RBF) is used to extend the small free surface deflection assumption by Blyth and Pozrikidis [1] through accurate determination of free surface quantities such as curvature and unit normal for larger surface deflections. For flow around cylinders incorporation of a contact angle condition within the RBF was required, and within a parameter study results for various size cylinders presented.

\section{Acknowledgements}

This research was carried out at the University Technology Centre in Gas Turbine Transmission Systems at the University of Nottingham with financial support from Rolls-Royce plc, Aerospace Group. The views expressed in this paper are those of the authors and not necessarily those of Rolls-Royce plc, Aerospace Group.

\section{References}

[1] Blyth, M.G. \& Pozrikidis, C., Film flow down an inclined plane over a threedimensional obstacle. Physics of Fluids, 18(5), pp. 052104-14, 2006.

[2] Hayes, M., O’Brien, G. \& Lammers, J.H., Greens function for steady flow over a small two-dimensional topography. Physics of Fluids, 12(11), pp. 28452858, 2000.

[3] Pozrikidis, C. \& Thoroddsen, S.T., The deformation of a liquid film flowing down an inclined plane wall over a small particle arrested on the wall. Physics of Fluids A, 3(11), pp. 2546-2558, 1991.

[4] Sellier, M., The flow of a thin liquid film past a cylinder. COMSOL Users Conference 2006, Frankfurt, pp. 151-154, 2006. 